\title{
Communications
}

\section{Concours pour l'obtention du prix de la Fondation Mogens et Wilhelm Ellermann}

En 2020, la Société Suisse de Neurologie attribuera pour la $17^{\mathrm{e}}$ fois le prix de la Fondation Mogens et Wilhelm Ellermann, d'un montant de 20000 CHF, qui est destiné à récompenser

a) un travail scientifique dans le domaine neurologique, qui a été publié lors des deux dernières années ou qui a été accepté pour publication par une revue reconnue sur le plan international;

b) une monographie qui n'a pas les caractéristiques d'un traité ni celles d'une revue de la littérature;

c) l'ensemble d'une œuvre scientifique dans le domaine des sciences neurologiques.
Ce travail doit avoir été rédigé par un ou plusieurs chercheurs suisses ou par des chercheurs étrangers en activité dans une clinique ou un institut helvétique. La préférence sera donnée à des auteurs jeunes qui ne sont pas agrégés ou ne le sont que depuis peu.

Les lettres de candidature accompagnées d'un CV et d'une liste des publications (seulement travaux qui sont peer-reviewed) doivent être adressées jusqu'au 31 janvier 2020 au Prof. Dr méd. C. L. Bassetti, Chef de service, Clinique et Policlinique neurologique, Hôpital universitaire de Berne, Inselspital, 3010 Berne. Veuillez envoyer votre candidature par e-mail à l'adresse suivante: irene.kaufmann[at]insel.ch 